\title{
Urolithiasis incidence in patients with dental plaque
}

\author{
Vlad Alin Pantea', Larisa Renata Pantea-Rosan², Teodor Maghiar ${ }^{3}$ \\ ${ }^{1}$ Department of Dental Medicine, Faculty of Medicine and Pharmacy, University of Oradea, Romania \\ ${ }^{2}$ Department of Medical Disciplines, Faculty of Medicine and Pharmacy, University of Oradea, Romania \\ ${ }^{3}$ Department of Morphologycal Disciplines, Faculty of Medicine and Pharmacy, University of Oradea, Romania
}

\begin{abstract}
Introduction. Kidney stones are more and more common among the population, and multiple physico-chemical elements, as well as numerous risk factors, lead to its occurrence. Dental plaque is found especially in patients with poor oral hygiene or a genetic predisposition and consists in the mineralization of bacterial plaque on the surface of the teeth or prosthetic works.

Material and method. The study enrolled 107 hospitalized patients who presented reno-urinary stones and / or dental plaque.

Results. Increased incidence of urolithiasis in terms of average age in women, while the incidence is higher in male patients over 60 years. Patients who also associated dental plaque showed an increase in the incidence of reno-urinary stones compared to those without tooth dental plaque, and women who had dental plaque showed a predisposition to urolithiasis compared to people without dental plaque.

Conclusions. There is a close link between the appearance of dental plaque and renolithiasis, many factors contributing equally to their detection, and people with dental plaque are more prone to the appearance of renolithiasis compared to those who do not have the disease.
\end{abstract}

Keywords: urolithiasis, dental plaque, reno-urinary stones, incidence

\section{INTRODUCTION}

Reno-urinary lithiasis is the most common condition found in the population with a condition of the reno-urinary tract. The factors that lead to its occurrence are multiple being influenced by the physico-chemical processes that take place at this level, eventually leading to the appearance of a set of mineral microelements that aggregate to form kidney stones (1).

Multiple elements that participate in the formation of kidney stones are also found in the formation of dental plaque. This is the result of mineralization of the bacterial plaque. It has been observed that, unlike kidney stones, which is more and more common, dental plaque correlates mainly with the demographic characteristics of the population, where in poorly developed countries the incidence of its occurrence is higher (2).

\section{OBJECTIVE}

The main objective of the study is to emphasize the connection between the occurrence of urolithiasis and dental plaque, being known that many risk factors are involved in both pathologies.

\section{MATERIAL AND METHOD}

We conducted a prospective study that enrolled 107 patients with urolithiasis and / or dental plaque, of which 50 were women and 57 were men. The patients were hospitalized in County Emergency Clinical Hospital of Oradea, Romania, between 01.01.2019 and 31.01.2020.

The study was conducted with the approval of the Ethics Committee of the Institution no 457/08.01.2019. 
The diagnosis of patients with urolithiasis was based on the correlation of clinical data with paraclinical data. The anamnestic data highlighted the presence of typical colic pain in the renal lodge that radiates to the inner face of the lower limb. The paraclinical data that significantly contributed to the diagnosis were made with the help of abdominal ultrasound, which in most cases could specify the presence of the horse at the renal or ureteral level.

The diagnosis of patients with dental plaque was made by simply inspecting the oral cavity.

In order to specify the dental plaque, both in the supragingival and the subgingival form, the consultation kit of the oral cavity was used during the inspection of the oral cavity by the dentist.

Abdominal ultrasound performed to objectify urolithiasis was performed using the Siemens Acusion X300 ultrasound.

As major criteria for inclusion in the study was the age of patients over 18 years and under 90 years, patients who agreed to participate in the study, patients who were diagnosed during hospitalization reno-urinary stones and / or or dental plaque.

Exclusion criteria were provided by patients who were not entitled to participate in the study, patients who had major mental or oncological disorders, patients who did not have urolithiasis and / or dental plaque.

Statistical analysis was performed using SPSS 20 and Microsoft Excel.

\section{RESULTS}

In women, the age was between 21 and 70 years, and in men between 28 and 72 years, the average age being insignificantly higher in women than in men (52.64 years vs. 51.67 years, $\mathrm{p}=0.622)($ table 1$)$.

TABLE 1. Distribution of uro-lithiasis patients by sex and age

\begin{tabular}{|l|c|c|c|c|}
\hline \multirow{2}{*}{\multicolumn{1}{|c|}{ Age group }} & \multicolumn{2}{c|}{ Women } & \multicolumn{2}{c|}{ Men } \\
\cline { 2 - 5 } & No. & $\%$ & No. & $\%$ \\
\hline$<30$ years & 3 & 6.00 & 4 & 7.02 \\
\hline 31-40 years & 8 & 16.00 & 8 & 14.04 \\
\hline $41-50$ years & 9 & 18.00 & 12 & 21.05 \\
\hline 51-60 years & 15 & 30.00 & 16 & 28.07 \\
\hline$>60$ years & 15 & 30.00 & 17 & 29.82 \\
\hline Average age (years) & \multicolumn{3}{|c|}{$52.64 \pm 10.30$} & \multicolumn{3}{c|}{$51.67 \pm 9.95$} \\
\hline
\end{tabular}

Nephrolithiasis was diagnosed in $33.64 \%$ of patients, the most common location being basinetal
$(25.23 \%)$. Ureterolithiasis was diagnosed in $55.14 \%$ of patients, and urinary bladder stones in $11.21 \%$ (table 2).

TABLE 2. Distribution of patients according to the location of kidney stones

\begin{tabular}{|l|c|c|}
\hline \multicolumn{1}{|c|}{ Diagnostic } & No. & \% \\
\hline Nephrolithiasis & 36 & 33,64 \\
\hline Pelvic & 27 & 25,23 \\
\hline Caliceal & 6 & 5,61 \\
\hline Coraliform & 3 & 2,80 \\
\hline Ureterolitiasis & 59 & 55,14 \\
\hline Bladder stones & 12 & 11,21 \\
\hline
\end{tabular}

In the group of patients with dental plaque, regardless of age, both among patients under 50 years and over this age, the prevalence of uro-lithiasis is higher than the group of patients without dental plaque (Figure 1).

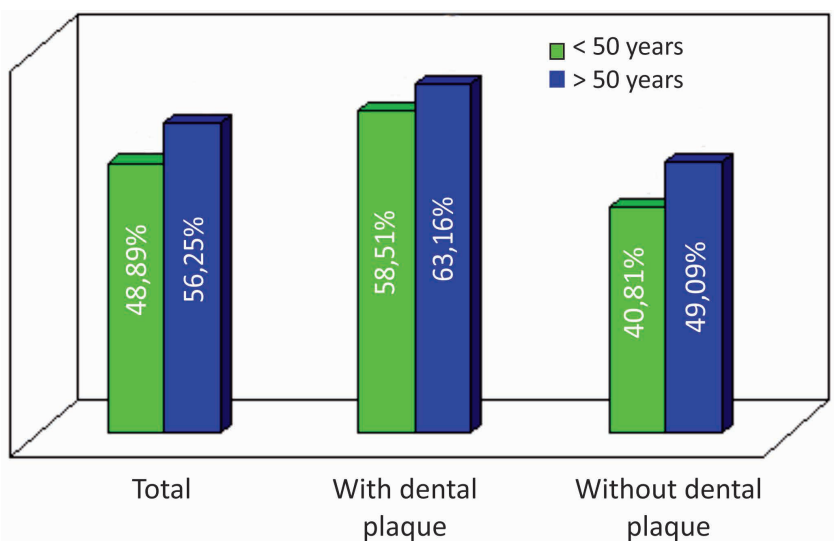

FIGURE 1. Prevalence of urolithiasis in patients with dental plaque in the elderly

An increased prevalence of urolithiasis regardless of the sex of the patients can also be observed in patients who have had dental plaque compared to those without (Figure 2).

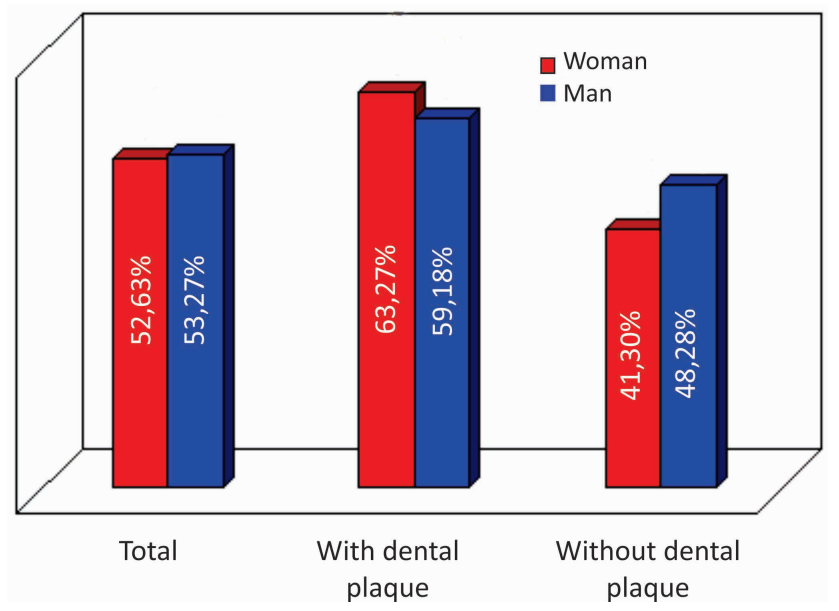

FIGURE 2. Prevalence of urolithiasis by sex 


\section{DISCUSSIONS}

In our study, 57 men and 50 women were enrolled, and the presence of urolithiasis was observed to be mainly higher among male patients both in the group of those under 50 years and in those over this age. Our data are consistent with other specialized studies that have reported an increased prevalence of nephrolithiasis in the male population (3).

In patients with nephrolithiasis, pelvic location was predominant in both the under-50 or older age group, while the location of bladder stones had a low incidence among patients, regardless of age.

Thus we can say that, regardless of the age or sex of patients, ureterolithiasis has an increased incidence among the population, compared to nephrotlithiasis, which predominates especially at the age of over 50 or bladder lithiasis.

Other authors also consider that ureteral localization of kidney stone is much more common in medical practice, compared to nephrolithiasis or urinary bladder stones, probably due to the fact that when ureterolithiasis is present most patients also have specific colic symptoms, given often by acute obstruction of the urinary system, forcing the patient to see a doctor (4).

It is known that any disorder in the oral cavity can resonate throughout the body, and the appearance of dental plaque can contribute to the detection of many pathologies. The mechanism of appearance and the contribution that dental tartar has

\section{REFERENCES}

1. Singh VK, Rai PK. Kidney stone analysis techniques and the role of major and trace elements on their pathogenesis: a review. Biophys Rev. 2014;6(3-4):291-310.

2. Sreenivasan PK, Prasad KVV, Javali SB. Oral health practices and prevalence of dental plaque and gingivitis among Indian adults. Clin Exp Dent Res. 2016;2(1):6-17.

3. Scales Jr CD, Smith AC, Hanley JM, Saigal CS. Prevalence of kidney stones in the United States. Eur Urol. 2012;62(1):160-5.

4. Chand RB, Shah AK, Pant DK, Paudel S. Common site of urinary calculi in kidney, ureter and bladder region. Nepal Med Coll J. 2013;15(1):5-7.

5. Marsh PD. Dental plaque as a biofilm and a microbial community implications for health and disease. BMC Oral Health. 2006; 6(Suppl 1):S14. in their appearance is more or less elucidated, depending on the pathology (5).

It has also been observed that patients over 50 years of age who have dental plaque have an increased incidence of developing urolithiasis. Aspects consistent with the literature in which there is an increased incidence among elderly patients with dental plaque, with a higher incidence of the incidence of nephrolithiasis in these patients, compared to those without dental plaque (6-8).

The strengths and novelties of this study are represented by research itself, because it is the first specialized article that addressed the two pathologies: kidney stones and dental plaque, and thus emphasizes the close connection that coexist between the two diseases. The limitation of the study are reflected by the small group of patients included in study and the short duration of time in which patients were monitored.

\section{CONCLUSIONS}

There is a close link between the appearance of dental plaque and renolithiasis, many factors contributing equally to their detection, and people with dental plaque are more prone to the appearance of renolithiasis compared to those who do not have the disease.

Consequently, patients who will have dental tartar may be referred to a urological examination or to perform a routine abdominal ultrasound to detect kidney stones.

Conflict of interest: none declared Financial support: none declared

6. Alexander RT, Hemmelgarn BR, Wiebe N, Bello A, Samuel S, Klarenbach SW, Curhan GC, Tonelli M; Kidney stones and cardiovascular events: A cohort study. CJASN. 2014;9:(3) 506-512.

7. Najafipour H, Mohammadi TM, Rahim F, Haghdoost AA, Shadkam $M$, Afshari M. Association of oral health and cardiovascular disease risk factors Results from a community based study on 5900 adult subjects. ISRN Cardiol. 2013;2013:782126.

8. Kati B, Kalkan E, Pelit ES, Yagmur I, Çiftçi H. Do dental calculi predict the presence of renal stones?. Arch Ital Urol Androl. 2018;90(3):159-162. 